\title{
Städtische Umwelt im Alter \\ Präferenzen älterer Menschen zum altersgerechten Wohnen, zur Wohnumfeld- und Quartiersgestaltung in Basel
}

\section{Rita Schneider-Sliwa, Basel}

\section{Einleitung}

Die Langlebigkeit und die Probleme einer alternden Gesellschaft gehören zu den grossen gesellschaftlichen Herausforderungen - auch in Basel: Im Jahr 2002 machten die über 60-jährigen im Kanton Basel-Stadt 49221 der 188115 Einwohner aus, also 26\%. Über 65-jährige machten rund 40000 Personen aus, und über 70-jährige stellten 29488 Personen bzw. 16\% der Bevölkerung. $90 \%$ aller über 80 -jährigen und $70 \%$ der über 90-jährigen lebten in Basel-Stadt eigenständig zu Hause in den eigenen vier Wänden.

Als Thema gehören Wohnen im Alter und der Bau von Altersheimen, Pflegeheimen, Seniorenzentren, Alten- und Behindertenwohnungen seit langem in die Zuständigkeit der öffentlichen Verwaltung. Allerdings sind die Aufgaben des zukünftigen Handelns in Sachen Wohnen im Alter keineswegs definiert oder altersgruppengerecht konzipiert (LaLIVE D'EPINAY et al. 1995). So gibt es im Kanton Basel-Stadt beispielsweise eine Alterspflegepolitik, welche konkrete Massnahmen nur für die hilfs- und pflegebedürftigen Personen - in der Regel Hochbetagte - ausrichtet. In Basel sind dies nur ca. 7\% der älteren Menschen. Für 93\% der über 65-jährigen, also mehr als 37000 ältere Menschen in Basel-Stadt, bleibt die generelle Frage relevant, wie sie ihre Eigenständigkeit im Alter im gewohnten Wohnumfeld erhalten wollen. An diesem Punkt setzt die vorliegende «Altersstudie» ein, die mit Unterstützung durch das Baudepartement des Kantons Basel-Stadt, Hauptabteilung Planung, den Seniorenverband Nordwestschweiz (SVNW), Pro Senectute Basel-Stadt, Baselland und Schweiz, die Novartis Pensionierten-Vereinigung, die Pensionskasse Hoffmann-La Roche, die Pensionskasse des Basler Staatspersonals, die Pensionskasse der Basler Kantonalbank sowie die Freiwillige Akademische Gesellschaft Basel und den Gallusser-Hausammann-Fonds entstand.

\section{Leitfragen und Zielsetzung}

Zwei Leitfragen wurde nachgegangen:

1. Welche Bedürfnisse haben ältere Menschen zwischen 65 und 85 Jahren, also die eigenständig Lebenden, bezüglich des Wohnens und Quartiers?
- Wie wollen die heutigen alten Menschen wohnen und leben?

- Was sind für sie altersgerechte Wohnformen?

- Wie wollen sie sich versorgen, welche Interessen und Kontakte pflegen?

2. Welche selbständigkeitsfördernden Massnahmen sind in den Bereichen Wohnraumversorgung, Quartiersgestaltung oder Sicherheit im öffentlichen Raum erwünscht?

- Welche Verbesserungen zur Gestaltung des Stadtraumes werden gewünscht?

- Welche Angebote für Seniorinnen und Senioren gibt es im Quartier? Wie werden diese genutzt?

Die Fragen zum Wohnen im Alter sind nicht gänzlich neu, ebenso wenig wie die zu erwartenden Antworten. Die Sichtweisen der älteren Menschen werden immerhin seit Jahren von Altersverbänden vertreten. Allerdings gab es bis anhin kaum breit abgestützte Meinungsbilder der älteren Menschen zum Wohnen im Alter. Die Studie ist die erste Grossbefragung von älteren Menschen in der Schweiz zum selbstbestimmten Wohnen im Alter (SCHNeIDER-SLIWA 2004).

Wohnen gehört zu den Grundbedürfnissen des Lebens und die eigenen vier Wände prägen weitgehend die Lebensqualität im Alter. Mit dem Wohnen verbindet sich die Möglichkeit, sein Leben selbständig und eigenverantwortlich zu gestalten. Planung für ein altengerechtes Umfeld darf daher nicht erst dann einsetzen, wenn ältere Menschen hilfs- und pflegebedürftig werden, sondern sie muss bereits zu einem früheren Zeitpunkt erfolgen. Die Weichen in Politik, Planung und vor allem der Immobilienwirtschaft sollten rechtzeitig dafür gestellt werden, dass ältere Menschen möglichst lange in ihrer vertrauten Umgebung bleiben können.

Die Studie wollte also durch ein breit abgestütztes Meinungsbild aus der Perspektive von fast dreieinhalbtausend Seniorinnen und Senioren einen Beitrag leisten:

- zur Diskussion in Basel um das Wohnen im Alter

- zur Weiterentwicklung von altersgerechten Wohnangeboten der Privatwirtschaft

- zur Bedürfnisgerechtigkeit, barrierefreiem Bauen und Wohnraumanpassung

- zur Weiterentwicklung traditioneller und neuer Wohnformen für ältere Menschen

- zum betreuten Wohnen, Service-Wohnen 
- zur Förderung der Partizipation von älteren Menschen bei strukturellen Entscheidungen im Wohnungsbau und in der Wohnungswirtschaft.

\section{Gedanklicher Hintergrund - Alter als neue Lebensphase}

Mensch-Sein ist keine Frage des Alters - der Mensch bleibt Bürger bis zum letzten Atemzug. Alter und alte Menschen unterscheiden sich jedoch von Generation zu Generation. Die heutigen «Alten» sind im Gegensatz zu ihrer Elterngeneration aktiv, hochmobil und erfolgreich: sie halten sich gesund, haben Kaufkraft, finanzieren ihre Familien, leisten soziale Dienste an Kindern, Enkeln und den eigenen Eltern sowie im Gemeinwesen. Bis in ihre achtziger Jahre leisten ältere Menschen in sozialer und wirtschaftlicher Hinsicht mehr für die Gesellschaft, als sie diese kosten. Für die Gesellschaft sind die «neuen Alten» ein unverzichtbarer Stützpfeiler und für die Wirtschaft ein wertvoller Kompetenzträger (KоHLI et al. 1993; KoHLI \& KüNEMUND 1996, 2000; KüNeMund 2000; HöPFLINGER 1992, 1997, 2001; HöPFLINGer \& STUCKeLBERGER 1999a).

«Das Alter» gibt es nicht mehr, wohl aber nuancierte Prozesse des Älterwerdens, bei denen deutlich differenzierte Lebensphasen und Altersstrukturen innerhalb des sogenannten Dritten oder Vierten Alters erkennbar sind (FrETER 1993; KÜNEMUND 2000; RedAYMulvey 2003; Ristau \& Mackroth 1993; PerrigChiello \& HöPflinger 2001; Rosenmayr \& Kolland 1988; SaAKe 1998; Weymann 2000; Widmer \& SousaPoza 2003; Willis \& ReID 1999). Interindividuelle Differenzen in den biologischen Funktionen sowie im Prozess des Alterns werden mit dem Alter grösser. Zwischen den Menschen höheren Alters bestehen hinsichtlich ihrer körperlichen und psychischen Funktionen und Fähigkeiten grössere Unterschiede als bei Jungen (Spiegel 2003: 13). Daher unterscheiden sich auch die Bedürfnisse der heutigen alten Menschen im Allgemeinen und innerhalb der unterschiedlichen Lebensphasen zwischen 65 und über 85 von denen der Vorgängergeneration. Wegen der gestiegenen Lebenserwartung und des hohen Grades an Eigenständigkeit, den die Älteren sich durch ihre eigene Lebensweise bewahren, ist es wichtig, dass die Älteren auch in der Gesellschaft eingebunden bleiben: die Integration ins Gemeindeleben, grösstmögliche Autonomie und Selbstbestimmung, Kontinuität in der Lebensführung in der gewohnten Umgebung - dies sind zunehmend die Themen, welche die Bedürfnisse der älteren Menschen auf einen gemeinsamen Nenner bringen.

Viele Untersuchungen zur Altersforschung wurden für die Schweiz im Rahmen des Nationalen Forschungspro- grammes (NFP 32) «Alter/Vieillesse/Anziani» (HöPFLinger \& STUCKelberger 1999b) erarbeitet (AREND \& HöPflinger 1997; WeHRLi-SChINDLer 1997). Das Wohnumfeld und das Wohnen im Alter wurden in der Schweiz jedoch noch zu wenig berücksichtigt, mehr Studien liegen aus dem deutschen Kontext vor (DeutsChes Zentrum für Altersfragen 1998, 2001; Grosshans 2001; Narten 1997; Petersen 1997; Blonski et al. 1997). Dass das Thema des eigenständigen Wohnens und der altersgerechten Wohnformen eine immer stärkere Bedeutung in der Gesellschaft erhalten muss, ist jedoch verständlich. Das eigene Leben bis zum Schluss selbst gestalten und an der gesellschaftlichen Entwicklung teilhaben zu können sind die Bedürfnisse, die sich hauptsächlich in der Wohnsituation widerspiegeln. Im Alltag bedeutet Eigenständigkeit im Alter eine altersgerechte, eigenständigkeitserhaltende Wohnform, den Ausbau von Versorgungsdiensten, welche das eigenständige Wohnen länger möglich machen, ferner angemessene Freizeit- und Bildungsangebote sowie Kontakte, Selbsthilfeeinrichtungen, Teilnahme am Leben im öffentlichen Raum, Mobilitätserhalt, Informations- und Beratungsdienste. Wohnen bleibt jedoch der zentralste Lebensbereich.

Die Sicht der älteren Menschen auf ihre Wohnbedürfnisse im Alter ist bislang in Basel und der Schweiz nicht durch eine gross angelegte Befragung ermittelt worden, obwohl

- sich Wohnbedürfnisse mit zunehmendem Alter ändern und durch eine spezifische Lebensgeschichte geprägt sind

- die alternde Gesellschaft die Privatwirtschaft und die öffentliche Verwaltung mit der Frage nach altersgerechter Wohnraumversorgung konfrontiert

- der Erhalt des selbständigen Lebens in der sozialen, städtischen und infrastrukturellen Umwelt die öffentlichen Ausgaben für eine alternde Gesellschaft wesentlich entlasten könnte.

Städtische Umwelt und Wohnen im Alter - dieser Fokus der Studie untersucht also ein Themenfeld, das einer gesamtgesellschaftlichen Offensive bedarf, denn Wohnen ist der sozial-strukturelle Rahmen des individuellen Lebens.

\section{Ergebnisse}

Von 7217 angesprochenen älteren Menschen, davon 389 im persönlichen Interview - durchgeführt durch Mitglieder des Nordwestschweizer Seniorenverbandes -, wurden 3248 gültige Fragebogen zurückgesandt (Rücklauf von 45.3\%). Von den 3248 Personen (94\% Schweizerinnen und Schweizer, 6\% andere Nationalitäten, vorwiegend Deutsche) waren 1862 $(57.3 \%)$ männlichen und 1314 (40.5\%) weiblichen 


\begin{tabular}{|l|r|r|r|r|r|r|}
\hline Derzeit alters- Altersgruppe & $\begin{array}{c}\text { 60-65 J. } \\
\text { in \% } \\
\text { gerechte Wohnung }\end{array}$ & $\begin{array}{c}\text { 66-70 J. } \\
\text { in \% } \\
(\mathrm{n}=820)\end{array}$ & $\begin{array}{c}\mathbf{7 1 - 7 5 ~ J .} \\
\text { in \% } \\
(\mathrm{n}=799)\end{array}$ & $\begin{array}{c}\mathbf{7 6 - 8 0} \text { J. } \\
\text { in \% } \\
(\mathrm{n}=559)\end{array}$ & $\begin{array}{c}\mathbf{8 1 - 8 5} \text { J. } \\
\text { in \% } \\
(\mathrm{n}=251)\end{array}$ & $\begin{array}{c}\text { ïber 85 J. } \\
\text { in \% } \\
(\mathrm{n}=30)\end{array}$ \\
\hline ja & 72.0 & 70.3 & 68.4 & 63.0 & 62.5 & 60.0 \\
zum Teil & 18.3 & 17.6 & 19.7 & 23.1 & 24.3 & 30.0 \\
nein & 7.9 & 10.3 & 9.8 & 11.4 & 11.6 & 6.7 \\
weiss nicht & 0.7 & 0.4 & 0.9 & 1.3 & 0.4 & 3.3 \\
k. A. & 1.1 & 1.4 & 1.2 & 1.2 & 1.2 & 0.0 \\
\hline Gesamt & 100.0 & 100.0 & 100.0 & 100.0 & 100.0 & 100.0 \\
\hline
\end{tabular}

Tab. 1: Derzeit altersgerechte Wohnung - nach Altersgruppen

Own home currently suitable for old age, by age group

Appartement actuel conforme à l'âge, par catégories d'âge

Quelle: SCHNEIDER-SLIWA 2004

Geschlechts. In einer «klassischen» Ehe- oder Partnerschaft ohne Kinder lebten $60.3 \%$ der Befragten, in einer Ehe bzw. Partnerschaft mit Kindern 3.9\%.64.2\% also sind das Zusammenleben in einer ehelichen/ partnerschaftlichen Solidargemeinschaft gewohnt. Mit Abstand die nächst grössere Gruppe war die der Alleinstehenden ohne Kinder mit 29.6\%. Die Strukturen des Samples sind im allgemeinen ausgeglichen und lassen nicht a priori Schieflagen im Meinungsbild entstehen.

\subsection{Altersgerechte Wohnung}

In Basel-Stadt empfinden $69.3 \%$ der Befragten und in Baselland $65.6 \%$ ihre Wohnung als derzeit altersgerecht und weitere hohe Anteile sehen dies partiell so $(19.1 \%$ in Basel-Stadt und $21.3 \%$ in Baselland). Dass ihre Wohnung in der Zukunft noch altersgerecht wäre, verneinen hohe Anteile entschieden. Nur noch $36.3 \%$ in Basel-Stadt und $29.7 \%$ in Baselland sehen ihre Wohnung auch in der Zukunft als altersgerecht an, wobei für $34.4 \%$ in Basel-Stadt und $41.9 \%$ in Baselland dies nur partiell zutrifft. Die Statistiken zeigen eine sachlich-nüchterne Einschätzung, dass die Wohnumstände in Zukunft nicht oder nur zum Teil ausreichend sein werden. Nach Altersgruppen aufgeschlüsselt zeigt sich (Tab. 1,2), dass die «jungen Alten» ihre Wohnungen mit $72 \%$ als deutlich besser geeignet für ihr Alter ansehen als die über 75-jährigen $(63 \%)$, die über 80 -jährigen $(62.5 \%)$ oder die über 85 -jährigen (60\%). Demgegenüber steigen die Anteile derer, die ihre Wohnung als nur zum Teil altersgerecht empfinden mit zunehmendem Alter $(17.6 \%$ bei den $66-$ bis 70 -jährigen, $30 \%$ bei den über 85 -jährigen). Dies liegt daran, dass die Befragten mit steigendem Alter ihre Wohnung bereits ihren Bedürfnissen sukzessive angepasst haben. Auffallend ist der mit dem Alter sin-

\begin{tabular}{|l|r|r|r|r|r|r|}
\hline Altersgruppe & $\begin{array}{c}\text { 60-65 J. } \\
\text { in Zukunft alters- } \\
\text { gerechte Wohnung }\end{array}$ & $\begin{array}{c}\text { 66-70 J. } \\
\text { in \% } \\
(\mathrm{n}=820)\end{array}$ & $\begin{array}{c}\mathbf{7 1 - 7 5} \mathbf{~ J .} \\
\text { in \% } \\
(\mathrm{n}=799)\end{array}$ & $\begin{array}{c}\mathbf{7 6 - 8 0} \mathbf{~ J .} \\
\text { in \% } \\
(\mathrm{n}=576)\end{array}$ & $\begin{array}{c}\mathbf{8 1 - 8 5} \mathbf{J} . \\
\text { in \% } \\
(\mathrm{n}=251)\end{array}$ & $\begin{array}{c}\text { über 85 J. } \\
\text { in \% } \\
(\mathrm{n}=30)\end{array}$ \\
\hline ja & 34.0 & 32.3 & 36.5 & 32.2 & 37.5 & 36.7 \\
zum Teil & 39.0 & 39.2 & 36.5 & 38.6 & 31.1 & 23.3 \\
nein & 20.7 & 23.7 & 21.0 & 21.6 & 20.3 & 23.3 \\
weiss nicht & 4.9 & 2.8 & 4.6 & 5.9 & 6.4 & 13.3 \\
k. A. & 1.4 & 2.0 & 1.4 & 1.7 & 4.7 & 3.4 \\
\hline Gesamt & 100.0 & 100.0 & 100.0 & 100.0 & 100.0 & 100.0 \\
\hline
\end{tabular}

Tab. 2: In Zukunft altersgerechte Wohnung - nach Altersgruppen

Own home in the future still considered suitable for old age, by age group

Appartement futur conforme à l'âge, par catégories d'âge

Quelle: SCHNEIDER-SLIWA 2004 


\begin{tabular}{|c|c|c|c|c|}
\hline Derzeit alters- $\begin{array}{c}\text { Monatsbrutto- } \\
\text { einkommen } \\
\text { gerechte Wohnung }\end{array}$ & $\begin{array}{c}\text { unter } 2^{\prime} \mathbf{5 0 0} \mathbf{F r} \text {. } \\
\text { in } \% \\
(\mathrm{n}=71)\end{array}$ & $\begin{array}{c}\mathbf{2}^{\prime} \mathbf{5 0 0}-\mathbf{5}^{\prime} \mathbf{0 0 0} \mathbf{F r} . \\
\text { in } \% \\
(\mathrm{n}=972)\end{array}$ & $\begin{array}{c}\mathbf{5}^{\prime} \mathbf{0 0 1}-\mathbf{7}^{\prime} \mathbf{5 0 0} \mathbf{F r} . \\
\text { in } \% \\
(\mathrm{n}=1016)\end{array}$ & $\begin{array}{c}\text { über } \mathbf{7}^{\prime} \mathbf{5 0 0} \mathbf{~ F r} \text {. } \\
\text { in } \% \\
(\mathrm{n}=739)\end{array}$ \\
\hline ja & 64.8 & 65.7 & 69.1 & 73.7 \\
\hline zum Teil & 15.5 & 22.1 & 19.8 & 16.8 \\
\hline nein & 15.5 & 10.1 & 9.5 & 7.8 \\
\hline weiss nicht & 1.4 & 1.0 & 0.5 & 0.3 \\
\hline k. A. & 2.8 & 1.1 & 1.1 & 1.4 \\
\hline Gesamt & 100.0 & 100.0 & 100.0 & 100.0 \\
\hline
\end{tabular}

Tab. 3: Derzeit altersgerechte Wohnung - nach Einkommensgruppen

Own home currently suitable for old age, by income class

Appartement actuel conforme à l'âge, par catégories de revenus

Quelle: SCHNEIDER-SLIWA 2004

kende Anteil derer, die ihre Wohnung nur noch als teilweise altersgerecht sehen, was darauf zurückzuführen ist, dass mit steigendem Alter «Zukunft» anders bewertet wird und die Realität der eigenen Wohnung besser erkannt wird.

$65.7 \%$ der Befragten in der Einkommensgruppe 2500 5000 Fr., jedoch $73.7 \%$ in der Einkommensgruppe über 7500 Fr. sehen ihre Wohnung derzeit als altersgerecht an, was mit ihren grösseren finanziellen Möglichkeiten zusammenhängen dürfte, ihre Wohnung tatsächlich jederzeit besser für sich verändernde Bedürfnisse auszurichten (Tab. 3, 4). Mit steigendem Einkommen steigt insgesamt der Anteil derer, die ihre Wohnung in der Zukunft für teilweise altersgerecht halten. Man kann ableiten, dass eine Nachfrage nach altersgerech- ten Wohnungen zu einem gewissen marktkonformen Preis und in einer gewissen Grösse vorhanden ist und die Wohnungsbauträger dies stärker berücksichtigen sollten.

\subsection{Wohnen in unterschiedlichen Wohnsituationen - Beispiel «Altersheim»}

Im Falle, dass man seine eigene Wohnung aufgeben müsste, besteht ein klarer Wunsch nach Wohnraum, der den Selbstständigkeitserhalt fördert und dem Bedarf sowie der modernen Lebensweise undWürde des alten Menschen gerecht wird. So wird an klassische «Altersheimsituationen» eine dezidierte Absage erteilt: In keiner Altersgruppe wird eine klassische Wohnraumversorgung mit Einzimmerwohnungen in Kollektivhaushalten gewünscht, sondern moderne, altersge-

\begin{tabular}{|c|c|c|c|c|}
\hline $\begin{array}{l}\text { In Zukunft alters- } \\
\text { gerechte Wohnung }\end{array}$ & $\begin{array}{c}\text { unter } \mathbf{2}^{\prime} \mathbf{5 0 0} \mathbf{F r} \text {. } \\
\text { in } \% \\
(\mathrm{n}=71)\end{array}$ & $\begin{array}{c}\mathbf{2}^{\prime} \mathbf{5 0 0}-\mathbf{5}^{\prime} \mathbf{0 0 0} \mathbf{F r} . \\
\text { in } \% \\
(\mathrm{n}=972)\end{array}$ & $\begin{array}{c}\mathbf{5}^{\prime} \mathbf{0 0 1}-\mathbf{7}^{\prime} \mathbf{5 0 0} \mathbf{~ F r} . \\
\text { in } \% \\
(\mathrm{n}=1016)\end{array}$ & $\begin{array}{c}\text { über } \mathbf{7}^{\prime} \mathbf{5 0 0} \mathbf{~ F r} \text {. } \\
\text { in } \% \\
(\mathrm{n}=739)\end{array}$ \\
\hline ja & 28.2 & 36.9 & 34.2 & 32.6 \\
\hline zum Teil & 38.0 & 33.2 & 38.8 & 42.2 \\
\hline nein & 26.8 & 22.3 & 21.4 & 19.4 \\
\hline weiss nicht & 4.2 & 4.9 & 4.1 & 4.5 \\
\hline k. A. & 2.8 & 2.7 & 1.5 & 1.3 \\
\hline Gesamt & 100.0 & 100.0 & 100.0 & 100.0 \\
\hline
\end{tabular}

Tab. 4: In Zukunft altersgerechte Wohnung - nach Einkommensgruppen Own home in the future still considered suitable for old age, by income class Appartement futur conforme à l'âge, par catégories de revenus Quelle: SCHNEIDER-SLIWA 2004 


\begin{tabular}{|l|r|r|r|r|r|r|r|r|}
\hline Altersgruppe & $\begin{array}{c}\text { 60-65 J. } \\
\text { in \% } \\
(\mathrm{n}=820)\end{array}$ & $\begin{array}{c}\text { 66-70 J. } \\
\text { in \% } \\
(\mathrm{n}=799)\end{array}$ & $\begin{array}{c}\mathbf{7 1 - 7 5} \text { J. } \\
\text { in \% } \\
(\mathrm{n}=776)\end{array}$ & $\begin{array}{c}\mathbf{7 6 - 8 0} \text { J. } \\
\text { in \% } \\
(\mathrm{n}=559)\end{array}$ & $\begin{array}{c}\mathbf{8 1 - 8 5} \mathbf{J} . \\
\text { in \% } \\
(\mathrm{n}=251)\end{array}$ & $\begin{array}{c}\text { iber 85 J. } \\
\text { in \% } \\
(\mathrm{n}=30)\end{array}$ & $\begin{array}{c}\text { k. A. } \\
\text { in \% } \\
(\mathrm{n}=36)\end{array}$ & $\begin{array}{c}\text { Gesamt } \\
\text { in \% } \\
(\mathrm{n}=3271)\end{array}$ \\
\hline ja & 7.7 & 6.9 & 7.5 & 6.8 & 6.8 & 3.3 & 5.6 & 7.2 \\
neingehörigen & 88.0 & 89.2 & 85.7 & 84.1 & 79.3 & 86.7 & 83.3 & 86.4 \\
k. A. & 4.3 & 3.9 & 6.8 & 9.1 & 13.9 & 10.0 & 11.1 & 6.4 \\
\hline Gesamt & 100.0 & 100.0 & 100.0 & 100.0 & 100.0 & 100.0 & 100.0 & 100.0 \\
\hline
\end{tabular}

Tab. 5: Wohnen bei Angehörigen - nach Altersgruppen Living with relatives, by age group

Habitation chez de la parenté, par catégories d'âge

Quelle: ScHNEIDER-SLIWA 2004

rechte Wohnungen in unterschiedlichen Grössen, in denen man seine Selbstständigkeit länger bewahren kann. In der Absage an diese althergebrachte «Altenunterbringung» sind sich beide Geschlechter einig, wobei Personen mit höheren Bildungsabschlüssen eine besonders deutliche Ablehnung zum Ausdruck bringen.

\subsection{Leben in der Wohnung eines Angehörigen}

Ein dezidiertes Nein wird auch dem Wohnen in der Wohnung/im Haus eines Angehörigen erteilt. 88.1\% in Basel-Stadt und $85.5 \%$ in Baselland wünschen keine Mitwohngelegenheit bei Verwandten, dies ist altersunabhängig (Tab. 5). Nach einem Jahrzehnte währenden eigenständigen Leben will man im Alter nicht den Familienangehörigen zur Last fallen oder auch nicht mehr in die Verantwortung für die Familien der eigenen Kinder eingebunden werden. Die Absage an das Wohnen mit Angehörigen ist nicht sehr stark einkommensgebunden (Tab. 6): Zwar ist der Anteil derer, die sich ein Zusammenleben mit den Angehörigen vorstellen könnten, mit $12.7 \%$ in der untersten Einkommensgruppe mehr als doppelt so hoch wie bei den Haushalten in der Höchstverdienergruppe mit $5.7 \%$ (über 7500 Fr.). $\mathrm{Zu}$ denken geben jedoch die insgesamt sehr hohen Anteile derer in allen Eink ommensgruppen, die unter allen Umständen unabhängig leben möchten. Diese Haltung ist nicht geschlechterspezifisch.

\subsection{Leben in einer Wohngemeinschaft mit Personen unterschiedlichen Alters}

Mit $85.5 \%$ spricht sich das Gesamtsample klar gegen ein Leben in einer Wohngemeinschaft aus. Angesichts der bestehenden Lebenssituation, in der der Grossteil in Partnerschaften lebt, ist eine Fortführung dieses Lebensmusters gewünscht: man will implizit die Beibehaltung des Gewohnten. Die Absage an diese Wohnform ist nicht altersabhängig (Tab.7). Die deutliche Mehrheit der Befragten möchte nicht in einer intergenerationellen Wohngemeinschaft leben, wobei sich nur leicht geschlechtsspezifische Unterschiede zeigen. Frauen, denen man i.A. eine höhere Sozialkompetenz attestiert, befürworten in etwas grösserem Masse mit

\begin{tabular}{|c|c|c|c|c|c|c|}
\hline $\begin{array}{l}\text { Einkommen } \\
\text { Umzug zu- } \\
\text { Angehörigen }\end{array}$ & $\begin{array}{c}\text { unter } 2^{\prime} 500 \mathrm{Fr} \text {. } \\
\text { in } \% \\
(\mathrm{n}=71)\end{array}$ & $\begin{array}{c}2^{\prime} 500-5^{\prime} \mathbf{0 0 0} \mathbf{~ F r} . \\
\text { in } \% \\
(\mathrm{n}=972)\end{array}$ & $\begin{array}{c}5^{\prime} \mathbf{0 0 1}-7^{\prime} \mathbf{5 0 0} \mathrm{Fr} . \\
\text { in } \% \\
(\mathrm{n}=1016)\end{array}$ & $\begin{array}{c}\text { uiber } 7 \mathbf{5 0 0} \mathbf{F r} \text {. } \\
\text { in } \% \\
(\mathrm{n}=739)\end{array}$ & $\begin{array}{c}\text { k. A. } \\
\text { in } \% \\
(n=473)\end{array}$ & $\begin{array}{c}\text { Gesamt } \\
\text { in \% } \\
(n=3271)\end{array}$ \\
\hline ja & 12.7 & 8.3 & 7.6 & 5.7 & 5.3 & 7.2 \\
\hline nein & 80.3 & 85.5 & 86.5 & 88.9 & 84.8 & 86.4 \\
\hline k. A. & 7.0 & 6.2 & 5.9 & 5.4 & 9.9 & 6.4 \\
\hline Gesamt & 100.0 & 100.0 & 100.0 & 100.0 & 100.0 & 100.0 \\
\hline
\end{tabular}

Tab. 6: Wohnen bei Angehörigen - nach Einkommensgruppen

Living with relatives, by income class

Habitation chez de la parenté, par catégories de revenus

Quelle: SchNEIDER-SLIWA 2004 


\begin{tabular}{|c|c|c|c|c|c|c|c|c|}
\hline $\begin{array}{l}\text { Altersgruppe } \\
\text { Umzug in } \\
\text { WG }\end{array}$ & $\begin{array}{c}60-65 \mathbf{J} \\
\text { in } \% \\
(n=820)\end{array}$ & $\begin{array}{c}66-70 \mathbf{J} \\
\text { in } \% \\
(n=799)\end{array}$ & $\begin{array}{c}\mathbf{7 1 - 7 5} \mathbf{J} \\
\text { in } \% \\
(\mathrm{n}=776)\end{array}$ & $\begin{array}{c}76-80 \mathbf{J} \\
\text { in } \% \\
(n=559)\end{array}$ & $\begin{array}{c}\mathbf{8 1 - 8 5} \mathbf{J} \\
\text { in } \% \\
(\mathrm{n}=251)\end{array}$ & $\begin{array}{c}\text { über } \mathbf{8 5} \mathbf{J} . \\
\text { in } \% \\
(\mathrm{n}=30)\end{array}$ & $\begin{array}{c}\text { k. A. } \\
\text { in } \% \\
(n=36)\end{array}$ & $\begin{array}{c}\text { Gesamt } \\
\text { in } \% \\
(n=3271)\end{array}$ \\
\hline $\mathrm{Ja}$ & 13.9 & 7.0 & 5.9 & 6.3 & 4.8 & 3.3 & 8.3 & 8.2 \\
\hline Nein & 81.8 & 89.1 & 87.2 & 84.6 & 81.3 & 86.7 & 80.6 & 85.4 \\
\hline k. A. & 4.3 & 3.9 & 6.9 & 9.1 & 13.9 & 10.0 & 11.1 & 6.4 \\
\hline Gesamt & 100.0 & 100.0 & 100.0 & 100.0 & 100.0 & 100.0 & 100.0 & 100.0 \\
\hline
\end{tabular}

Tab. 7: Umzug in altersgemische Wohngemeinschaft - nach Altersgruppen

Moving to mixed age communal residences, by age group

Déménagement dans une communauté résidentielle d'âge mixte, par catégories d'âge

Quelle: SCHNEIDER-SLIWA 2004

11.1\% diese Lebensform (Tab. 8). Die Nichtpräferenz für eine intergenerationelle Wohngemeinschaft zeigt einen minimalen Einkommenseffekt (Tab. 9):Während $15.5 \%$ der untersten Einkommensgruppen sich diese Wohnform vorstellen können, sind es nur $7.6 \%$ der Höchstverdiener. Mit dem Einkommen steigt die Ablehnung solcher Wohnformen: sind es $77.5 \%$ der finanzschwächeren Haushalte, die sich gegen eine Wohngemeinschaft aussprechen, sind es bei den Höchstverdienern schon $87.0 \%$. Allerdings darf man dem Einkommenseffekt keine allzu grosse Bedeutung beimessen: Immerhin ist die Ablehnung bei allen Einkommensgruppen eindeutig sehr hoch, was dafür spricht, dass man sich im Raum Basel bei der Suche nach angemessenen Wohnformen für alte Menschen nicht auf die Schiene der Wohngemeinschaften begeben sollte. Wohngemeinschaften werden als alternative Lebensform an sich abgelehnt, was vor dem Hintergrund gesehen werden muss, dass die Befragten grösstenteils in langjährigen Paarbeziehungen leben und eine Wohngemeinschaft eine Paarbeziehung weder ersetzt noch ihr ein angemessenes Umfeld bietet.

\subsection{Leben in einer Wohngemeinschaft mit Betagten}

In die gleiche Richtung weisen die Ergebnisse zum Wohnen in einer Wohngemeinschaft mit Betagten. Bei der Aufgabe der eigenen Wohnung aus Altersgründen würden ca. 90\% der Befragten, also 2928 Personen, keinesfalls an Möglichkeiten denken, die in der öffentlichen Diskussion immer wieder geäussert werden - nämlich das Zusammenleben in Wohngemeinschaften von alten Menschen. Eine Wohngemeinschaft mit anderen alten Menschen entspricht derzeit jedenfalls ebenso wenig dem Lebensentwurf für das Alter wie die intergenerationelle Wohngemeinschaft (Tab. 10,11). $92.6 \%$ der 66 - bis 70 -jährigen und $82.5 \%$ der 81- bis 85-jährigen lehnen dies ab, wobei die Ablehnung mit steigendem Alter sinkt, aber dennoch sehr hoch bleibt $(80.0 \%)$. Auch wird dies von $90 \%$ der Personen in allen Einkommensgruppen und sowohl von Männern als auch Frauen mit 89.6\% bzw. 90.9\% schlichtweg abgelehnt.

Zwischenfazit: Weder die intergenerationelle noch die Wohngemeinschaft mit anderen Betagten halten ca. $90 \%$ der Befragten, immerhin über 2900 Personen, für

\begin{tabular}{|c|c|c|c|c|}
\hline $\begin{array}{l}\text { Umzug } \\
\text { in WG }\end{array}$ & $\begin{array}{c}\text { männlich } \\
\text { in } \% \\
(\mathrm{n}=1870)\end{array}$ & $\begin{array}{c}\text { weiblich } \\
\text { in } \% \\
(n=1324)\end{array}$ & $\begin{array}{c}\text { k. A. } \\
\text { in } \% \\
(\mathrm{n}=77)\end{array}$ & $\begin{array}{c}\text { Gesamt } \\
\text { In } \% \\
(n=3271)\end{array}$ \\
\hline $\mathrm{Ja}$ & 6.1 & 11.1 & 6.5 & 8.2 \\
\hline Nein & 87.2 & 83.1 & 80.5 & 85.4 \\
\hline k. A. & 6.7 & 5.8 & 13.0 & 6.4 \\
\hline Gesamt & 100.0 & 100.0 & 100.0 & 100.0 \\
\hline
\end{tabular}

Tab. 8: Umzug in altersgemischte Wohngemeinschaft - nach Geschlecht Moving to mixed age communal residences, by gender 


\begin{tabular}{|c|c|c|c|c|c|c|}
\hline $\begin{array}{l}\text { Emzug } \\
\text { in WG }\end{array}$ & $\begin{array}{c}\text { unter } \mathbf{2}^{\prime} \mathbf{5 0 0} \mathbf{F r} \text {. } \\
\text { in } \% \\
(\mathrm{n}=71)\end{array}$ & $\begin{array}{c}\mathbf{2}^{\prime} \mathbf{5 0 0}-\mathbf{5}^{\prime} \mathbf{0 0 0} \mathbf{F r} . \\
\text { in } \% \\
(\mathrm{n}=972)\end{array}$ & $\begin{array}{c}\mathbf{5}^{\prime} \mathbf{0 0 1}-\mathbf{7}^{\prime} \mathbf{5 0 0} \mathbf{F r} . \\
\text { in } \% \\
(\mathrm{n}=1016)\end{array}$ & $\begin{array}{c}\text { über } 7 \mathbf{5 0 0} \mathbf{F r} \text {. } \\
\text { in } \% \\
(\mathrm{n}=739)\end{array}$ & $\begin{array}{c}\text { k. A. } \\
\text { in } \% \\
(n=473)\end{array}$ & $\begin{array}{c}\text { Gesamt } \\
\text { in } \% \\
(n=3271)\end{array}$ \\
\hline $\mathrm{Ja}$ & 15.5 & 9.2 & 8.3 & 7.6 & 5.7 & 8.2 \\
\hline Nein & 77.5 & 84.7 & 85.8 & 87.0 & 84.4 & 85.4 \\
\hline k. A. & 7.0 & 6.1 & 5.9 & 5.4 & 9.9 & 6.4 \\
\hline Gesamt & 100.0 & 100.0 & 100.0 & 100.0 & 100.0 & 100.0 \\
\hline
\end{tabular}

Tab. 9: Umzug in altersgemischte Wohngemeinschaft - nach Einkommensgruppen Moving to mixed age communal residences, by income class Déménagement dans une communauté résidentielle d'âge mixte, par catégories de revenus Quelle: SCHNEIDER-SLIWA 2004

die geeignete Lebensform. Dies sollte deutliche Implikationen für die zukünftige Wohnraumversorgung für alte Menschen haben: Während das Leben im Generationenverbund heutzutage häufig in der politischen Arena als moderne neue Lebensform für ältere Menschen zum Nutzen von jung und alt gehandelt wird, ist dies eine Sichtweise, die derzeit eindeutig nicht der Vorstellung alter Menschen entspricht. Umgekehrt sollte man sich auch über die deutliche Ablehnung von Wohngemeinschaften mit Betagten Gedanken machen: Altersheime nach herkömmlichem Muster sind nichts anderes als Kollektivhaushalte mit Betagten, die heute kaum mehr Akzeptanz in der alternden Gesellschaft finden.

\subsection{Wohnpräferenzen bei Aufgabe der jetzigen Wohnung aus Altersgründen}

Es zeigt sich eine klare Absage an Klein- und Kleinstwohnungen im Alter, wenn man seine derzeitig grössere Wohnung aus Altersgründen aufgeben müsste. Die eindeutige Präferenz für 2- bis 21/2-Zimmer-Alterswohnungen - betreut und unbetreut - ist bedingt durch die Partnersituation und den Wunsch nach Bewahrung des gelebten Lebensmusters, der Lebensqualität und der Eigenständigkeit als Paar. Gewünscht werden betreute, rollstuhlgängige Wohnsituationen (Tab. 12,13), wobei die Grösse der Wohnung nicht unter 2⿺辶/2 Zimmern liegen sollte (Tab. 14, 15, 16).

\section{7 $\mathrm{Zu}$ den Vorstellungen der Seniorinnen und Senioren von altersgerechten Wohnformen}

Wohnformen, in denen der Raum zu klein ist, um mit dem Partner zu leben, Gäste zu bewirten und einen eigenen kleinen Haushalt zu bewirtschaften, sind inakzeptabel. Wohnformen, in denen nur ein eigener Raum zurVerfügung steht und andere Räumlichkeiten geteilt werden müssen, sei das in einer Heimsituation oder in einer Wohngemeinschaft, sind inakzeptabel. «Wohnen im Alter»- das bedeutet auch in erster Linie, das Wohnen im Quartier zu fördern. Über 50\% der Personen wohnen länger als 20 Jahre am gleichen Ort im Quartier/in der Gemeinde. Dem Quartier resp. der Gemeinde kommt daher eine grosse Bedeutung und Symbolik in einer affektiv-emotionalen und kogni-

\begin{tabular}{|c|c|c|c|c|c|c|c|c|}
\hline $\begin{array}{l}\text { Altersgruppe } \\
\text { Wohnen } \\
\text { in WG mit } \\
\text { Betagten }\end{array}$ & $\begin{array}{c}60-\mathbf{6 5} \mathbf{J} \\
\text { in } \% \\
(\mathrm{n}=820)\end{array}$ & $\begin{array}{c}\text { 66-70 J. } \\
\text { in \% } \\
(\mathrm{n}=799)\end{array}$ & $\begin{array}{c}\mathbf{7 1 - 7 5} \mathbf{J} \\
\text { in } \% \\
(\mathrm{n}=776)\end{array}$ & $\begin{array}{c}\mathbf{7 6 - 8 0} \mathbf{J} . \\
\text { in \% } \\
(\mathrm{n}=559)\end{array}$ & $\begin{array}{c}\mathbf{8 1 - 8 5} \mathbf{J} . \\
\text { in \% } \\
(\mathrm{n}=251)\end{array}$ & $\begin{array}{c}\text { über } \\
\mathbf{8 5} \mathbf{J} . \\
\text { in \% } \\
(n=30)\end{array}$ & $\begin{array}{c}\text { k. A. } \\
\text { in } \% \\
(n=36)\end{array}$ & $\begin{array}{c}\text { Gesamt } \\
\text { in } \% \\
(n=3271)\end{array}$ \\
\hline $\mathrm{Ja}$ & 4.3 & 3.5 & 2.6 & 3.6 & 3.6 & 10.0 & 2.8 & 3.5 \\
\hline Nein & 91.5 & 92.6 & 90.6 & 87.3 & 82.5 & 80.0 & 86.1 & 90.0 \\
\hline k. A. & 4.2 & 3.9 & 6.8 & 9.1 & 13.9 & 10.0 & 11.1 & 6.5 \\
\hline Gesamt & 100.0 & 100.0 & 100.0 & 100.0 & 100.0 & 100.0 & 100.0 & 100.0 \\
\hline
\end{tabular}

Tab. 10: Wohngemeinschaft mit Betagten - nach Altersgruppen Communal residence for elderly people, by age group Communauté résidentielle pour personnes âgées, par catégories d'âge Quelle: SchNEIDER-SLIwA 2004 


\begin{tabular}{|c|c|c|c|c|c|c|}
\hline $\begin{array}{l}\text { Wohnen } \\
\text { in WG mit } \\
\text { Betagten }\end{array}$ & $\begin{array}{c}\text { unter } 2^{\prime} \mathbf{5 0 0} \mathbf{F r} \text {. } \\
\text { in } \% \\
(\mathrm{n}=71)\end{array}$ & $\begin{array}{c}\mathbf{2}^{\prime} \mathbf{5 0 0}-\mathbf{5}^{\prime} \mathbf{0 0 0} \mathbf{~ F r} . \\
\text { in } \% \\
(\mathrm{n}=972)\end{array}$ & $\begin{array}{c}\mathbf{5}^{\prime} \mathbf{0 0 1}-\mathbf{7}^{\prime} \mathbf{5 0 0} \mathbf{~ F r} . \\
\text { in } \% \\
(\mathrm{n}=1016)\end{array}$ & $\begin{array}{c}\text { über } 7^{\prime} \mathbf{5 0 0} \mathbf{F r} \text {. } \\
\text { in } \% \\
(n=739)\end{array}$ & $\begin{array}{c}\text { k. } \mathbf{A} . \\
\text { in } \% \\
(n=473)\end{array}$ & $\begin{array}{c}\text { Gesamt } \\
\text { in \% } \\
(n=3271)\end{array}$ \\
\hline $\mathrm{Ja}$ & 2.8 & 4.7 & 3.9 & 2.4 & 2.1 & 3.5 \\
\hline Nein & 90.1 & 89.1 & 90.2 & 92.2 & 87.9 & 90.0 \\
\hline k. A. & 7.1 & 6.2 & 5.9 & 5.4 & 10.0 & 6.5 \\
\hline Gesamt & 100.0 & 100.0 & 100.0 & 100.0 & 100.0 & 100.0 \\
\hline
\end{tabular}

Tab. 11: Wohngemeinschaft mit Betagten - nach Einkommensgruppen

Communal residence for elderly people, by income class

Communauté résidentielle pour personnes âgées, par catégories de revenus

Quelle: SCHNEIDER-SLIWA 2004

tiv erkannten Beziehung zu. Eine sozial nachhaltige, altersgerechte Planung ist daher eine Stadt- und Quartiersentwicklung mit einem starken Fokus auf modernen, altersgerechten, möglichst betreuten, mindestens 21/2-Zimmer grossen Wohnungen im Quartier; ferner der Ausbau versorgerischer Leistungen zum Verbleib im angestammten Wohnraum.

\subsection{Wichtigste Faktoren bei Aufgabe der Wohnung aus Altersgrïinden}

Wichtigste Ergebnisse: Angestrebt wird die Selbständigkeit bis ins hohe Alter. Das Verbleiben in der eigenen Wohnung mit Unterstützung durch die Spitex ist wichtig $(85.2 \%)$, die eigene Familie/Kinder sollen möglichst nicht belastet werden. Der Bedarf an betreuten und unbetreuten Alterswohnungen ist gross, nicht aber der an Pflegewohngruppen. Im Ernstfall will man sich auf Fachpersonal verlassen können, Betreuungsformen, die jedoch Abhängigkeiten schaffen (Angehörige, Freunde und Bekannte) werden klar weniger oder gar nicht als wichtig erachtet und damit implizit abgelehnt. Geschätzt wird im Bedarfsfall ein Mahlzeitenservice, der als bezahlte Leistung die Eigenständigkeit der Person nicht in Frage stellt. Institutionalisierte Hilfestellungen geniessen eine breitere Akzeptanz als persönliche. Insgesamt ergibt sich ein Ranking nach Spitex-Dienst, Familienangehörigen, Freunden, Nachbarschaftshilfe, wobei der SpitexDienst mit Abstand das wichtigste Hilfemodell ist. Nach Alter zeigt sich eine geringer werdende Akzeptanz für gewisse Hilfeleistungen, entweder, weil man erkennt, dass diese im Bedarfsfall nicht mehr ausreichen würden oder weil man soweit ohne solche Hilfestellungen ausgekommen ist und sich diese auch in der Zukunft nicht für sich denken kann.

\section{Fazit}

Das Meinungsbild spiegelt nicht nur Meinungen, sondern das Leben und die Lebensführung der so genannten Altengeneration. Die Resultate sind ein deutli-

\begin{tabular}{|l|r|r|r|r|r|r|r|r|}
\hline $\begin{array}{l}\text { Altersgruppe } \\
\text { Betreutes }\end{array}$ & $\begin{array}{c}\text { 60-65 J. } \\
\text { in \% } \\
\text { Wohnen }\end{array}$ & $\begin{array}{c}\text { 66-70 J. } \\
\text { in \% } \\
(\mathrm{n}=820)\end{array}$ & $\begin{array}{c}\mathbf{7 1 - 7 5} \mathbf{~ J .} \\
\text { in \% } \\
(\mathrm{n}=799)\end{array}$ & $\begin{array}{c}\mathbf{7 6 - 8 0 ~ J . ~} \\
\text { in \% } \\
(\mathrm{n}=559)\end{array}$ & $\begin{array}{c}\mathbf{8 1 - 8 5} \mathbf{~ J .} \\
\text { in \% } \\
(\mathrm{n}=251)\end{array}$ & $\begin{array}{c}\text { iuber 85 J. } \\
\text { in \% } \\
(\mathrm{n}=30)\end{array}$ & $\begin{array}{c}\text { k. A. } \\
\text { in \% } \\
(\mathrm{n}=36)\end{array}$ & $\begin{array}{c}\text { Gesamt } \\
\text { in \% } \\
(\mathrm{n}=3271)\end{array}$ \\
\hline Ja & 50.2 & 52.7 & 53.1 & 46.0 & 39.8 & 40.0 & 50.0 & 49.9 \\
Nein & 45.5 & 43.6 & 40.1 & 45.1 & 46.2 & 50.0 & 38.9 & 43.7 \\
k. A. & 4.3 & 3.7 & 6.8 & 8.9 & 14.0 & 10.0 & 11.1 & 6.4 \\
\hline Gesamt & 100.0 & 100.0 & 100.0 & 100.0 & 100.0 & 100.0 & 100.0 & 100.0 \\
\hline
\end{tabular}

Tab. 12: Wohnen in betreuter, rollstuhlgängier Alterswohnung - nach Altersgruppen

Living in wheel-chair accessible, sheltered old age apartments, by age group

Habitation dans un logement pour personnes âgées, disposant des services à la personne et accessible par chaise roulante, par catégories d'âge

Quelle: SCHNEIDER-SLIWA 2004 


\begin{tabular}{|c|c|c|c|c|c|c|}
\hline $\begin{array}{l}\text { Einkom- } \\
\text { Betreutes } \\
\text { Wohnen }\end{array}$ & $\begin{array}{c}\text { unter } 2 \text { '500 Fr. } \\
\text { in } \% \\
(\mathrm{n}=71)\end{array}$ & $\begin{array}{c}2^{\prime} 500-5^{\prime} 000 \text { Fr. } \\
\text { in } \% \\
(n=972)\end{array}$ & $\begin{array}{c}5^{\prime} 001-7^{\prime} 500 \mathrm{Fr} . \\
\text { in } \% \\
(\mathrm{n}=1016)\end{array}$ & $\begin{array}{c}\text { über } 7 \text { '500 Fr. } \\
\text { in } \% \\
(\mathrm{n}=739)\end{array}$ & $\begin{array}{c}\text { k. } \mathbf{A} . \\
\text { in } \% \\
(n=473)\end{array}$ & $\begin{array}{c}\text { Gesamt } \\
\text { in } \% \\
(n=3271)\end{array}$ \\
\hline $\mathrm{Ja}$ & 49.3 & 49.1 & 52.3 & 50.1 & 46.3 & 49.9 \\
\hline Nein & 43.7 & 44.8 & 41.9 & 44.7 & 43.8 & 43.7 \\
\hline k. A. & 7.0 & 6.1 & 5.8 & 5.2 & 9.9 & 6.4 \\
\hline Gesamt & 100.0 & 100.0 & 100.0 & 100.0 & 100.0 & 100.0 \\
\hline
\end{tabular}

Tab. 13: Wohnen in betreuter, rollstuhlgängiger Alterswohnung - nach Einkommensgruppen Living in wheel-chair accessible, sheltered old age apartments, by income class

Habitation dans un logement pour personnes âgées, disposant des services à la personne et accessible par chaise roulante, par catégories d'âge

Quelle: SCHNEIDER-SLIWA 2004

\begin{tabular}{|c|c|c|c|c|c|c|c|}
\hline Anz. Zimmer & $\begin{array}{c}\text { 60-65 J. } \\
\text { in \% } \\
(n=397)\end{array}$ & $\begin{array}{c}\text { 66-70 J. } \\
\text { in \% } \\
(\mathrm{n}=390)\end{array}$ & $\begin{array}{c}\text { 71-75 J. } \\
\text { in \% } \\
(n=378)\end{array}$ & $\begin{array}{c}76-80 \mathrm{~J} \\
\text { in } \% \\
(n=239)\end{array}$ & $\begin{array}{c}\text { 81-85 J. } \\
\text { in } \% \\
(n=81)\end{array}$ & $\begin{array}{c}\text { über } 85 \mathbf{J} . \\
\text { in } \% \\
(\mathrm{n}=12)\end{array}$ & $\begin{array}{c}\text { Gesamt } \\
\text { in \% } \\
(n=1497)\end{array}$ \\
\hline 1-1 1/2 Zimmer & 8.3 & 11.5 & 13.8 & 17.6 & 21.0 & 50.0 & 12.9 \\
\hline 2-2 1/2 Zimmer & 58.4 & 55.4 & 60.8 & 58.6 & 53.1 & 33.3 & 57.8 \\
\hline 3-3 1/2 Zimmer & 29.7 & 30.5 & 22.8 & 19.7 & 23.5 & 16.7 & 26.1 \\
\hline 4 und mehr Zimmer & 3.6 & 2.6 & 2.6 & 4.1 & 2.4 & 0.0 & 3.2 \\
\hline Gesamt & 100.0 & 100.0 & 100.0 & 100.0 & 100.0 & 100.0 & 100.0 \\
\hline
\end{tabular}

Tab. 14: Anzahl gewünschter Zimmer bei Wohnen in betreuter, rollstuhlgängier Alterswohnung - nach Altersgruppen

Number of rooms desired by residents of wheel-chair accessible, sheltered old age apartments, by age group

Nombre de chambres souhaitées dans un immeuble pour personnes âgées, à logements disposant des services à la personne et accessible par chaise roulante, par catégories d'âge

Quelle: SCHNEIDER-SLIWA 2004

\begin{tabular}{|c|c|c|c|c|c|c|}
\hline Anz. Zimmer & $\begin{array}{c}\text { unter } 2^{\prime} 500 \mathbf{F r} \text {. } \\
\text { in } \% \\
(\mathrm{n}=29)\end{array}$ & $\begin{array}{c}2^{\prime} \mathbf{5 0 0}-\mathbf{5}^{\prime} \mathbf{0 0 0} \mathbf{F r} . \\
\text { in } \% \\
(\mathrm{n}=449)\end{array}$ & $\begin{array}{c}\mathbf{5}^{\prime} \mathbf{0 0 1}-\mathbf{7}^{\prime} \mathbf{5 0 0} \mathbf{~ F r} . \\
\text { in } \% \\
(\mathrm{n}=487)\end{array}$ & $\begin{array}{c}\text { über } 7 \mathbf{7 0 0} \mathbf{~ F r} \text {. } \\
\text { in } \% \\
(\mathrm{n}=351)\end{array}$ & $\begin{array}{c}\text { k. A. } \\
\text { in \% } \\
(n=199)\end{array}$ & $\begin{array}{c}\text { Gesamt } \\
\text { in } \% \\
(n=1515)\end{array}$ \\
\hline 1-1 1/2 Zimmer & 37.9 & 21.8 & 9.7 & 3.4 & 14.1 & 12.9 \\
\hline 2-2 1/2 Zimmer & 51.7 & 63.9 & 66.3 & 39.9 & 55.8 & 57.8 \\
\hline 3-3 1/2 Zimmer & 10.4 & 13.4 & 23.4 & 48.1 & 25.1 & 26.1 \\
\hline 4 und mehr Zimmer & 0.0 & 0.9 & 0.6 & 8.6 & 5.0 & 3.2 \\
\hline Gesamt & 100.0 & 100.0 & 100.0 & 100.0 & 100.0 & 100.0 \\
\hline
\end{tabular}

Tab. 15: Anzahl gewünschter Zimmer bei Wohnen in betreuter, rollstuhlgängiger Alterswohnung - nach Einkommensgruppen

Number of rooms desired by residents of wheel-chair accessible, sheltered old age apartments, by income class

Nombre de chambres souhaitées dans un immeuble pour personnes âgées, disposant des services à la personne et accessible par chaise roulante, par catégories de revenus

Quelle: SCHNEIDER-SLIWA 2004 


\begin{tabular}{|l|r|r|r|r|}
\hline Geschlecht & $\begin{array}{c}\text { Männlich } \\
\text { in \% } \\
(\mathrm{n}=791)\end{array}$ & $\begin{array}{c}\text { Weiblich } \\
\text { in \% } \\
(\mathrm{n}=695)\end{array}$ & $\begin{array}{c}\text { k. A. } \\
\text { in \% } \\
(\mathrm{n}=29)\end{array}$ & \multicolumn{2}{c|}{$\begin{array}{c}\text { Gesamt } \\
\text { in \% } \\
(\mathrm{n}=1515)\end{array}$} \\
\hline 1-1 1/2 Zimmer & 9.7 & 16.5 & 13.8 & 12.9 \\
2-2 1/2 Zimmer & 54.2 & 61.6 & 65.5 & 57.8 \\
3-3 1/2 Zimmer & 32.4 & 19.4 & 17.2 & 26.1 \\
4 und mehr Zimmer & 3.7 & 2.5 & 3.5 & 3.2 \\
\hline Gesamt & 100.0 & 100.0 & 100.0 & 100.0 \\
\hline
\end{tabular}

Tab. 16: Anzahl gewünschter Zimmer bei Wohnen in betreuter, rollstuhlgängiger Alterswohnung - nach Geschlecht

Number of rooms desired by residents of wheel-chair accessible, sheltered old age apartments, by gender

Nombre de chambres souhaitées dans un immeuble pour personnes âgées, disposant des services à la personne et accessible par chaise roulante, par sexe

Quelle: SCHNEIDER-SLIWA 2004

ches Plädoyer für eine individuelle Lebensführung nach gewohntem, aber altersgerechtem Muster. Der Lebensentwurf als Paar in einer angemessen grossen Umgebung, die ein würdevolles Leben nach jahrzehntelangem Habitus zulässt, zeigt sich in der Absage an Treffpunkte für Gemeinschaftspflege und in der Bejahung des vertrauten sozialen Umfelds, wozu auch die Wohnungsgrösse gehört, die die Eigenständigkeit und das Verbleiben in den gewohnten Lebensumständen mit dem Partner sichert. Als Gesamtfazit der Studie ergibt sich, dass die Klischeevorstellung von den «Alten» als Gruppe, die sich nicht mehr selbst organisieren kann und für die Versorgungseinrichtungen geschaffen werden müssen, gründlich revidiert werden muss - mit Auswirkungen auf die Planung: Eine stärkere Mitgestaltungsorientierung der Seniorinnen und Senioren in der (zur Zeit kaum existierenden) amtlichen Alterspolitik in den beiden Basel ist eindeutig notwendig; ferner eine Abkehr von der dominanten Angebotsseite im Wohnungsmarkt aus Sicht der Anbieter (Bauwirtschaft, Bewirtschaftung der heutigen Immobilienbestände) und Planer hin zu einer nachfrageorientierten Sicht der betroffenen älteren Menschen.

\section{Literatur}

Arend, M. \& F. Höpflinger (1997): Zur Wohnversorgung und Wohnsituation älterer Menschen in der Schweiz. - Nationales Forschungsprogramm 32 «Alter», Zürich: Schweizerischer Nationalfonds zur Förderung der wissenschaftlichen Forschung.

BLonski, H. et al. (1997): Wohnformen im Alter: ein Praxisratgeber für die Altenhilfe. - Weinheim: Beltz Verlag.

Deutsches Zentrum Für Altersfragen (1998): Wohnen im Alter: Zweiter Bericht zur Lage der älteren Generation in der Bundesrepublik Deutschland. - Berlin.

Deutsches Zentrum für Altersfragen (2001): Alter und Gesellschaft: Dritter Bericht zur Lage der älteren
Generation in der Bundesrepublik Deutschland. Berlin.

FreTER, H.-J. (1993): Vergesellschaftung durch Teilzeitarbeit. - In: KoнLI, M. et al. (Hrsg.): Engagement im Ruhestand: Rentner zwischen Erwerb, Ehrenamt und Hobby. - Opladen: Leske + Budrich: 59-108.

Grosshans, H. (2001): Wohnumfeld und Quartiersgestaltung: für das Wohnen im Alter im Generationenverbund. - Stuttgart: Fraunhofer IRB Verlag.

HöPfLINGER, F. (1992): Alter und Altersforschung in der Schweiz. - Zürich: Seismo Verlag.

Höpflinger, F. (1997): Bevölkerungssoziologie: Eine Einführung in bevölkerungssoziologische Ansätze und demographische Prozesse. - Weinheim, München: Juventa Verlag.

Höpflinger, F. (2001): Altsein in der Schweiz - Entwicklung und Gegenwart. - In: Pro Senectute Schweiz (Hrsg.): Unterwegs zu einer Gesellschaft des langen Lebens, Band 10: Hat die Solidarität der Generationen Zukunft? - Zürich: Pro Senectute Fachverlag.

Höpflinger, F. \& A. Stuckelberger (1999a): Demographische Alterung und individuelles Altern: Ergebnisse aus dem nationalen Forschungsprogramm «Alter/ Vieillesse/Anziani». - Zürich: Seismo Verlag.

Höpflinger, F. \& A. StuCKelberger, in Zusammenarbeit mit H.-M. Hagmann (1999b): Alter/Anziani/ Vieillesse. Hauptergebnisse und Folgerungen aus dem Nationalen Forschungsprogramm NFP32. - Bern: s.n.

KoHLI, M. \& H. KüNEMUND (1996): Nachberufliche Tätigkeitsfelder: Konzepte, Forschungslage, Empirie. - Schriftenreihe des Bundesministeriums für Familie, Senioren, Frauen und Jugend 130.1, Stuttgart: Verlag W. Kohlhammer.

Kohli, M. \& H. Künemund (2000): Die Grenzen des Alters - Strukturen und Bedeutung. - In: Perrig-Chiello, P. \& F. Höpflinger (Hrsg.): Jenseits des Zenits: Frauen und Männer in der zweiten Lebenshälfte. - Bern: Haupt Verlag: 37-60. 
KoHLI, M. et al. (1993): Engagement im Ruhestand: Rentner zwischen Erwerb, Ehrenamt und Hobby. Opladen: Leske + Budrich.

Künemund, H. (2000): «Produktive» Tätigkeiten. In: Kohli, M. \& H. Künemund (Hrsg.): Die zweite Lebenshälfte: Gesellschaftliche Lage und Partizipation im Spiegel des Alters-Survey. - Opladen: Leske + Budrich: 277-317.

Lalive D'EpinaY, C. et al. (1995): Altern in der Schweiz. Bilanz und Perspektiven. - Bericht der eidgenössischen Kommission, Bern: Eidgenössische Drucksachen- und Materialzentrale.

NARTEN, R. (1997): Wohnbedürfnisse alter, allein stehender Frauen. - In: Blonski, H. et al. (Hrsg.): Wohnformen im Alter: Ein Praxisratgeber für die Altenhilfe. - Weinheim: Beltz Verlag: 31-49.

Perrig-Chiello, P. \& F. Höpflinger (2001): Zwischen den Generationen: Frauen und Männer im mittleren Lebensalter. - Zürich: Seismo Verlag.

Petersen, U. (1997): Idee und Praxis gemeinschaftlicher Wohnformen im Alter - Wohnen im Wandel. - In: Blonski, H. et al. (Hrsg.): Wohnformen im Alter: ein Praxisratgeber für die Altenhilfe. - Weinheim: Beltz Verlag: 134-154.

Reday-Mulvey, G. (2003): Von der Politik des frühzeitigen Ruhestandes zur Förderung eines längeren Erwerbslebens. - Zürich: Avenir Suisse.

Ristau, M. \& P. Maскroth (1993): Produktivität eines neuen Alters: Lebensziele, Märkte und Produkte. - In: KLose, H.-U. (Hrsg.): Altern hat Zukunft: Bevölkerungsentwicklung und dynamische Wirtschaft. - Opladen: Westdeutscher Verlag: 222-250.

Rosenmayr, L. \& F. Kolland (Hrsg.) (1988): Arbeit - Freizeit - Lebenszeit: Grundlagenforschungen zu Übergängen im Lebenszyklus. - Opladen: Westdeutscher Verlag: 29-56.

SAAKE, I. (1998): Theorien über das Alter: Perspektiven einer konstruktivistischen Alternsforschung. - Opladen: Westdeutscher Verlag.

Schneider-Sliwa, R. (2004): Städtische Umwelt im Alter. Präferenzen älterer Menschen zum altersgerechten Wohnen, zur Wohnumfeld- und Quartiersgestaltung. $-=$ Basler Stadt- und Regionalforschung 26, Basel: Geographisches Institut der Universität.

SpIEgEL, R. (2003): AHV-Alter 67: Nicht nur eine ökonomische Frage. - In: Basler Zeitung vom 29.9.2003: 13.

WehrLi-Schindler, B. (1997): Wohnen im Alter. Zwischen Zuhause und Heim: braucht es neue Wohnformen für Betagte? - Zürich: Seismo Verlag.

Weymann, A. (2000): Sozialer Wandel, Generationsverhältnisse und Technikgenerationen. - In: KoHLI, M. \& M. SzYdLIK (Hrsg.): Generationen in Familie und Gesellschaft. - Opladen: Leske + Budrich:36-58.

Widmer, R. \& A. Sousa-Poza (2003): Verbreitung und Potenzial der Alters(teilzeit)arbeit in der Schweiz. Zürich: Avenir Suisse.
Willis, S. \& J. ReID (1999): Middle Age: New Thoughts, New Directions. - In: Willis, S. \& J. ReID (Hrsg.): Life in the Middle: Psychological and Social Development in Middle Age. - San Diego: Academic Press: 275-280.

\section{Zusammenfassung: Städtische Umwelt im Alter. Präferenzen älterer Menschen zum altersgerechten Wohnen, zur Wohnumfeld- und Quartiersgestaltung in Basel}

Wohnbedingungen sind nicht nur direkt, sondern auch indirekt für die Erfüllung vieler menschlicher Grundbedürfnisse in allen Phasen des Lebens für ein sinnerfülltes, eigenverantwortliches Leben Grundvoraussetzung. Zentrales Element einer Alterspolitik muss daher sein, Wohnformen zu fördern, die nicht nur das Raumbedürfnis älterer Menschen berücksichtigen, sondern auch ihren Wunsch nach Sicherheit und Betreuung durch entsprechende Dienstleistungen.

Diese Grossbefragung von 3248 Personen zwischen 65 bis 85 Jahren in den Kantonen Basel-Stadt und Baselland ermittelte, wie die heutigen «Alten» wohnen und leben (wollen), was für sie altersgerechte Wohnformen sind, wie sie sich versorgen (wollen), welche mobilitätsfördernden, selbständigkeitserhaltenden Massnahmen sie im Wohnumfeld, Quartier, Stadtraum wünschen und welche zukunftsorientierten Veränderungen im Wohnumfeld, Quartier, Stadtraum die eigenständige Selbstversorgung gewährleisten können.

Es zeigt sich eine klare Absage an Klein- und Kleinstwohnungen im Alter, wenn man seine derzeitig grössere Wohnung aus Altersgründen aufgeben müsste. Die eindeutige Präferenz für 2-2 $1 \frac{1}{2}$-Zimmer-Alterswohnungen - betreut und unbetreut - ist bedingt durch die Partnersituation und den Wunsch nach Bewahrung des gelebten Lebensmusters, der Lebensqualität und der Eigenständigkeit als Paar. Die Resultate sind ein deutliches Plädoyer für eine individuelle Lebensführung nach gewohntem, aber altersgerechtem Muster. Der Lebensentwurf als Paar in einer angemessen grossen Umgebung, die ein würdevolles Leben nach jahrzehntelangem Habitus zulässt, zeigt sich in der Absage an Treffpunkte für Gemeinschaftspflege und in der Bejahung des vertrauten sozialen Umfelds, wozu auch die Wohnungsgrösse gehört, die die Eigenständigkeit und das Verbleiben in den gewohnten Lebensumständen mit dem Partner sichert. Das Meinungsbild spiegelt nicht nur Meinungen, sondern das Leben und die Lebensführung der so genannten Altengeneration. Wegen der tatsächlichen unabhängigen Lebensführung, dem Wunsch nach einer Fortführung des gewohnten Lebensmusters, der Tatsache, dass eigenständige Lebensführung preiswerter für die Gesellschaft ist und wegen dem Respekt vor dem Menschen und dem Gebot der sozialen Nachhaltigkeit sind 
alle Massnahmen auszubauen, die der Mobilität, Versorgung (z.B. Einkaufsmöglichkeiten, Quartiersläden) und dem Erhalt des Wohnumfelds bzw. einer adäquaten Wohnraumversorgung im Alter dienlich sind.

\section{Summary: The Urban Environment in Old Age. Pref- erences of senior citizens concerning the design of residential environment and locality in Basel}

Housing conditions are a fundamental precondition for the satisfaction of many basic human needs in all phases of life if we want to continue leading a meaningful, personally responsible life in old age. Promoting forms of housing that not only take into account the senior citizen's need for space, but also his or her wishes for security and care through the provision of appropriate services must therefore be a key element in policies dealing with old age.

This survey of 3248 persons aged 65 to 85 years in the Cantons of Basel-City and Basel-Country, Switzerland addressed the questions of how the senior citizen today is or wishes to be housed and live, what he or she views as forms of housing suitable for the senior citizen, how he or she cares or wishes to care for him/ herself, what measures he or she would like to see to promote mobility and maintain independence in the residential environment, locality, urban district, and what future-oriented changes in the residential environment, locality and urban district could assure independent self-care.

The results represent a clear plea of the elderly to be able to carry on living independently in the habitual way, but adapted for old age. Models for old age provision should give more consideration to alternative forms of housing, namely sheltered and unsheltered $2-2 \frac{1}{2}$ room flats. There is also a need for a stronger focus on and development of care services to help people stay in the home or residential area where they have lived for a long time. The desire to live as a couple in a sufficiently spacious environment, permitting a high quality of life such as has been enjoyed for decades, is reflected in the rejection of centres for communal care and a preference for the familiar social environment, the size of the living space in part guaranteeing independence and the opportunity to remain together with the partner. The results reflect not only the opinions but the whole life and lifestyle of the so-called older generation. Because of the current independent lifestyle of senior citizens and their desire to continue in familiar ways, the fact that independent is more valued in society than dependence, the need for respect of the individual and the importance of social cohesion, all possible measures should be taken to ensure mobility, provision of services (e.g. opportunities for shopping, local shops), preservation of housing arrangements and/or sufficient living space in old age.

\section{Résumé: L'environnement urbain pour le troisième âge. Choix des personnes âgées au regard du façonne- ment de l'environnement résidentiel et du quartier à Bâle}

Les conditions d'habitation ne sont pas seulement un préalable fondamental direct, mais aussi indirect pour l'accomplissement de nombreuses exigences humaines de base dans toutes les phases de l'existence, en vue de la pleine réalisation d'une vie responsable, qui donne du sens. C'est pourquoi toute politique en faveur du troisième âge doit être centrée sur la promotion de formes résidentielles adéquates, qui ne tiennent pas uniquement compte des besoins en espace des personnes âgées, mais aussi de la nécessité d'une garantie de sécurité et de services appropriés.

La présente investigation de grande ampleur auprès de 3248 personnes âgées de 65 à 85 ans, dans les cantons de Bâle Ville et de Bâle Campagne, a permis de savoir comment les personnes âgées habitent ou souhaitent habiter et vivre aujourd'hui, comment elles conçoivent les formes d'habitation adaptées à leur âge, de quelle façon elles s'approvisionnent ou souhaitent s'approvisionner, quelles mesures de promotion de la mobilité et du maintien de l'autonomie elles souhaitent pour leur environnement résidentiel, leur quartier et leur espace urbain. L'enquête a aussi révélé quelles transformations l'autonomie individuelle est à même de susciter à terme dans l'environnement résidentiel, le quartier et l'espace urbain.

La recherche montre de façon claire le refus d'un petit ou très petit logement, dès lors que les personnes âgées doivent renoncer pour des raisons d'âge à un logement plus grand. La préférence va indubitablement à un logement pour troisième âge de 2 à $2 \frac{1}{2}$ pièces, qu'il comporte des services à la personne ou non. Ce choix est motivé par la vie de couple et le désir de conserver le modèle du vécu, de la qualité de vie et de l'indépendance en tant que couple. Les réponses recueillies constituent un plaidoyer clair en faveur d'une vie autonome conforme au modèle de vie vécu jusqu'à présent, mais adapté aux nécessités de l'âge. Le projet de vie de couple, développé dans un environnement adapté, d'une certaine ampleur, qui permet de vivre une existence digne après des décennies de bien-être, s'exprime par le refus d'intégration dans des lieux de soins communautaires et la demande de maintien dans un environnement social familier, ce qui implique une taille résidentielle suffisamment grande, garante de l'autonomie et du maintien des conditions de vie habituelles avec le partenaire. L'image de l'opinion ne reflète pas seulement des opinions, mais la vie et la conduite de vie de la soi-disant génération de vieux. C'est à cause de la conduite de vie réellement indépendante, du désir de poursuite du modèle de vie habituel, du fait qu'une conduite de vie autonome est 
plus rentable pour la société, de même que du respect humain et de l'impératif d'une base sociale durable, qu'il importe de mettre en œuvre toutes les mesures susceptibles de favoriser la mobilité, l'approvisionnement (p.ex. possibilités d'achats, points de commerce de quartier) et le maintien de l'environnement résidentiel, respectivement de potentialités résidentielles adéquates pour la population âgée.

\section{Didaktische Hinweise}

- Welche Rolle spielen ältere Menschen (die «neuen Alten») als Stützpfeiler für die Gesellschaft und als Kompetenzträger in der Wirtschaft?

- In welcher Weise kann das individuelle Altern die Bedeutung des kollektiven Alterns schwinden lassen?

- Warum kann auch angesichts der steigenden Zahl der Hochbetagten das private und stationäre Wohnen im Pflegefall kein zentrales Thema einer Alterspolitik sein?

- Warum gehören Wohnzufriedenheit, Wohnbedürfnisse, Wohnwünsche und Wohnvorstellungen zu den wichtigsten Kriterien für die Evaluierung der Lebensqualität?
- Warum werden weder die intergenerationelle Wohngemeinschaft («Leben im Generationenverbund») noch die Wohngemeinschaft mit anderen Betagten als eine geeignete Lebensform im Alter erachtet?

- Welche Implikationen für die Wohnraumversorgung für alte Menschen hat dies?

Prof. Dr. Rita Schneider-Sliwa, Geographisches Institut der Universität Basel, Abteilung Humangeographie / Stadt- und Regionalforschung, Klingelbergstrasse 27, CH-4056 Basel, Schweiz.

e-mail: Rita.Schneider-Sliwa@unibas.ch

Manuskripteingang/received/manuscrit entré le 20.9.2004

Annahme zum Druck/accepted for publication/accepté pour l'impression: 1.12.2004 